\title{
General practitioners' perspectives on promoting sexual health to young men
}

Archibald Collyer, Siobhan Bourke, Meredith Temple-Smith

\section{Background and objectives \\ General practitioners (GPs) are well placed to promote sexual health to young men. Our previous research has suggested that this population expect GPs to initiate sexual health promotion. Little is known of GPs' perceptions of their role in sexual health promotion and perceived needs of young men.}

\section{Methods}

Semi-structured interviews with GPs were recorded until data saturation. Data were managed with NVivo; consensus was reached on thematic analysis.

\section{Results}

In 17 semi-structured interviews, a young man symptomatic with a sexually transmissible infection (STI) was the most common sexual health presentation. GPs identified a range of barriers to, and facilitators, of initiating discussions about sexual health. Some GPs reported no young male sexual health presentations. GPs generally believed young men should be taking more responsibility for their sexual health.

\section{Discussion}

Only some GPs endorsed young men's expectations that the GP would initiate a sexual health discussion. Increased awareness that young men are unlikely to seek sexual health advice would allow GPs to better tailor their approaches and increase opportunistic testing and sexual health promotion.
YOUNG AUSTRALIAN MEN are vulnerable in relation to their sexual health. Men aged 18-25 years use condoms inconsistently or not at all ${ }^{1}$ and engage in risky sexual behaviours at a higher rate than females in this age group, increasing their exposure to sexually transmissible infections (STIs) and potentially causing unintended pregnancy. ${ }^{2}$ Despite this, sexual and reproductive health promotion aimed at young men has received little attention.

Australian general practitioners (GPs) are well placed to provide sexual health promotion, as STIs are usually diagnosed in a general practice setting. ${ }^{3}$ However, young men attend general practice less frequently than young women. In 2016, of all presentations to general practice, $4.9 \%$ were young women aged 15-25 years, compared with $3 \%$ of young men in this age group. ${ }^{4}$

Our earlier research has shown young men lack knowledge of chlamydia and thus have unrealistic ideas about their risk of acquiring it..$^{5-7}$ This has also been shown in a UK study that found men were indifferent to chlamydia health promotion campaigns as they thought it was a women's disease. ${ }^{8}$ This may explain why young men will accept the offer of chlamydia testing less frequently than young women. ${ }^{9}$

Unless they experience worrying symptoms of an STI, young men are unlikely to seek sexual health advice. ${ }^{5}$ The asymptomatic nature of many STIs means that young men may be unaware of the need for medical care.

Young men generally want more sexual health information, ${ }^{7}$ and those aged 16-19 years identified primary care as their preferred setting for this. ${ }^{2}$ Our research showed that men aged 16-25 years would like GPs to initiate sexual health conversations with them even if they are not presenting with a problem relating to sexual health. ${ }^{5}$

No previous study has looked specifically at Australian GPs' perspectives on their role in promoting sexual health to young men. Little is known about the factors that would facilitate a GP's initiation of this sensitive discussion. To date, most research about GPs' sexual health practices has focused on STI testing rather than sexual health promotion. In 2003, an Australian study of men of all ages ${ }^{10}$ showed GPs' attitudes to and perspectives on sexual health may influence their counselling, assessment and management. This suggests that if sexual health is not a prominent issue for GPs, it is unlikely to be raised. Barriers previously identified to the likelihood of GPs raising sexual health include lack of time, practitioner's gender, and young men's infrequency, compared with young women, of presenting to a GP. ${ }^{11,12}$

US studies recommended annual sexual health consultations with young men, ${ }^{11}$ although GPs find it difficult to discuss sexual matters. ${ }^{12}$ In Australia, guidelines for preventive health are outlined in The Royal Australian College of General Practitioners' Guidelines for preventive activities in general practice (Red Book) ${ }^{13}$ and include the need to provide annual screening to young men and what constitutes a sexual health consultation. There is, however, no advice on initiation of a sexual health consultation.

To determine possible training needs for current and future GPs on how to overcome perceived barriers, we explored the perspectives of Victorian GPs on promoting sexual health to young men aged 16-25 years. 


\section{Methods}

This study used a qualitative design to explore GPs' perspectives on promoting sexual health to young men. Ethics approval was granted by the University of Melbourne (HREC 1545631.1).

\section{Participants}

A purposive sampling framework was used to ensure a variety of different perspectives. GPs from rural, regional and urban areas were recruited between February and May 2016. ${ }^{14} \mathrm{GPs}$ ' interest in sexual health and GPs' gender also guided recruitment. GP recruitment was conducted in three ways: at the Victorian Primary Care Practice-Based Research Network (VicReN) quarterly meeting following an oral presentation, through an advertisement placed in the VicReN newsletter, and by SB through her contacts in the Centre for Excellence in Rural Sexual Health (CERSH). Those interested in participating emailed AC, a male final-year medical student who had conducted previous research on young men and their understanding of sexual health. Snowballing, an established method in qualitative research in which participants were asked whether they had any acquaintances who fitted the study population, was also used. ${ }^{15}$

\section{Data collection}

Demographic data included how often GPs saw young men, time in practice, qualifications, age and practice size. The semi-structured interview guide comprised questions identified from the literature and prompted GPs to talk about sexual health consultations generally, young men's sexual health needs, whether GPs feel they promote sexual health, barriers faced by GPs when promoting sexual health and strategies used by GPs to overcome these. Interviews were conducted by AC and held at the GPs' convenience; all interviews were recorded. Data saturation was reached after 12 interviews, but sampling continued, to ensure representation of GPs from all of the sampling framework.

\section{Data analysis}

A qualitative data software package (NVivo) assisted in data management. The first seven interviews were transcribed verbatim and coded. Once coding was clear, the remaining 10 interviews were coded directly on the audio using NVivo, a practice becoming widely used to reduce the number of interpretative stages. ${ }^{16}$ Inductive reasoning was used to identify conceptual patterns and relationships in these codes in order to identify themes. A thematic coding framework was developed iteratively. Analyst triangulation ensured consensus on the themes was reached.

\section{Results}

Table 1 shows participant demographics. Interviews were 30-70 minutes, with a mean length of 50 minutes. Twelve interviews were conducted at clinic (five), home (two) or the University of Melbourne (five); five were conducted by phone.

During analysis, six themes were identified:

- Experiences of sexual health consultations showed GPs' prior experiences of discussing sexual health with young men.

- Risk assessment described how GPs made decisions around sexual health risk.

- Barriers to promoting sexual health with young men highlighted the barriers GPs faced when attempting to initiate sexual health discussion.

- Characteristics of young men highlighted GPs' preconceptions about young men's behaviour.

- Facilitators to a sexual health discussion described some GPs' tips for successful sexual health discussion with young men.

- Responsibility for initiating sexual health discussion described how GPs perceive their role in promoting sexual health to young men.

Selected illustrative quotes are presented in the text along with GP' self-selected pseudonyms. Quotes also disclose GPs' gender, age and self-declared interest in sexual health $(\mathrm{SH}+)$ or lack of interest (SH-).

\section{Experiences of sexual health consultations}

Young men were seen as a difficult group to discuss sexual health with and considered to be infrequent presenters, even if the GP was seeing a young male patient at least once a day.

It's tough getting a young man to come into your clinic, let alone discussing sexual health with them. (Amanda, female, 57 years, $\mathrm{SH}+$ )

Symptomatic young men occasionally initiated a sexual health discussion.

... when young men present, they usually have a specific issue. (Boston, female, 61 years, $\mathrm{SH}+$ )

Occasionally, young men would present for an asymptomatic STI screen.

Not routinely, but ... it's something that's becoming more and more common, more routine. (Matt, male, 45 years, $\mathrm{SH}-$ )

Opportunistic screening was used by many GPs.

I tend to grab the opportunity to talk to them about varied things. Because they don't see me as frequently as women (see me). (Aqua, female, 52 years, $\mathrm{SH}+$ )

GPs were aware that they needed to screen asymptomatic patients, but sometimes struggled to introduce sexual health into an unrelated presentation.

You don't have that easy segue, unless you're talking about something urological or bowel or bladder. (Brian, male 36 years, $\mathrm{SH}+$ )

They found this easier with women.

If someone comes in for the pill, it's easier ... you can sort of segue into it, without feeling as uncomfortable. (CP, male 33 years, $\mathrm{SH}-$ )

Some GPs with an interest in sexual health, however, had no difficulty introducing sexual health into the consultation. 
It doesn't take much effort on my behalf to get the conversation around ... to sexual health. (Jill, female, 30 years, $\mathrm{SH}+$ )

This contrasted with other GPs, particularly those who had no interest in sexual health.

I'd be below average, I'd imagine, because I don't do it very often. (Trish, female, 57 years, $\mathrm{SH}-$ )

\section{Risk assessment}

Conducting a risk assessment with a young man helped GPs to determine whether it was necessary to go further than screening for chlamydia.

... if we've found they had chlamydia, we would always use that as a flag to talk about sexual health, talk about how they may have got it last time, are they taking precautions to reduce the risk now. (Dr Grey, male, 59 years, $\mathrm{SH}+$ )

Some GPs did not attempt to measure a young man's risk, making the assumption that he was safe if using contraception.

I'm probably not interested in the number of partners they have, if they are using contraception. (Aqua, female, 52 years, $\mathrm{SH}-)$

\section{Barriers to promoting sexual health with young men}

GPs identified several barriers limiting their promotion of sexual health to young men: young men's reluctance to seek care, time constraints and gender discordance.

Young men not presenting to their practices, even when sick, was seen as a major barrier.

\section{Young men tend not to come to the doctor, as a cohort. Men in general tend to ruminate and self-diagnose, or ignore, and often only come in as a last resort. \\ (Dr Bob, male, 58 years, $\mathrm{SH}-$ )}

Once at the GP's, short consultation times offered little opportunity to discuss anything other than the presenting complaint.
So, they've come in for something else, they've got a 15-minute appointment, and you are usually running half an hour late - why would I open up a can of worms? (Barb, female, 51 years, $\mathrm{SH}-$ )

Older female GPs felt young men may not be comfortable with someone of their age.

With a female GP my age, for some of the younger guys, it might be like talking to their mum, and that would make it a bit uncomfortable. (Jule, female, 50 years, $\mathrm{SH}+$ )

Male GPs, in contrast, were happy to discuss sexual health with young men.

Because I think they get less put off with it if another man asks them. From that aspect it's less awkward. (Brian, male, 36 years, $\mathrm{SH}+$ )

\section{Characteristics of young men}

Most GPs were aware that young men's lack of knowledge made them vulnerable in relation to sexual health.

They're much less concerned about there being any STIs. (Kate, female, 32 years, $\mathrm{SH}+$ )

He probably has chlamydia; the fact (is) that they probably don't know ... that that stuff is treatable. (Brian, male, 36 years, $\mathrm{SH}+$ )

GPs suggested that those who were most embarrassed about their sexual health possibly would not present for sexual health advice. Typically, young men were seen as being difficult to engage.

Some young men really will not give you any information, whatsoever. They're the stereotypical teenage boy who just puts the wall up when you talk about anything to do with that. (Kate, female, 32 years, $\mathrm{SH}+$ )

Some of them you feel like you are banging your head against a wall doing a condom talk. Others are incredibly receptive. (Jill, female, 30 years, $\mathrm{SH}+$ )

\section{Facilitators to sexual health discussion}

GPs with a special interest in sexual health suggested the need to develop rapport before beginning a sexual health discussion.

If they know that I'm not uncomfortable talking about it, they are generally totally fine. (Brian, male, 36 years, $\mathrm{SH}+$ )

GPs who used technology were aware of internet resources available to help facilitate the sexual health discussion.

I guess there's the resources you can lean someone towards as well, the Melbourne Sexual Health website. (CP, male, 33 years, $\mathrm{SH}-)$

Others made sexual health a part of their clinic and normal practice. This included the use of posters in waiting rooms.

The posters around really remind people, 'Ohyeah, it has been a while since my last check'. (Jill, female, 30 years, $\mathrm{SH}+$ )

GPs believed that the Red Book did not go into enough detail when discussing young men's sexual health.

No, I don't think there's much ... I think there certainly should be some more in the Red Book. (Kate, female, 32 years, $\mathrm{SH}+$ )

\section{Responsibility to raise sexual health discussion}

There was a range of opinion around who should initiate discussion of sexual health. Some GPs felt it was their role.

... I don't know of any other person who would take responsibility. (Indiana, female, 58 years, $\mathrm{SH}+$ )

I see it as my role to bring up. (Barb, female, 51 years, $\mathrm{SH}^{-}$)

However, many GPs felt there was mixed responsibility in initiating a sexual health consultation.

I think it is the GP's to a point, but I think that it is the young man's responsibility primarily. (Kate, female, 32 years, $\mathrm{SH}+$ ) 
They also highlighted the need for young men to be more responsible regarding their sexual health.

[They] have a responsibility as well, and ... there are things that should be checked regularly if they have multiple partners. (Miranda, female, 57 years, $\mathrm{SH}-$ )

Several GPs deliberately made time to promote preventive sexual health.

I recommend it to every young man who comes in to see me. (Amanda, female, 57 years, $\mathrm{SH}+$ )

Most GPs mentioned that participation in this research would prompt them to include sexual health promotion in future consultations with young men.

I feel I should be asking some of these things a lot more. (Boston, female, 61 years, $\mathrm{SH}+$ )

\section{Discussion}

This study, the first of its kind, used semistructured interviews to explore Victorian GPs' attitudes around sexual health promotion to young men. GPs reported mixed experiences with sexual health consultations with young men. They were generally comfortable in dealing with young men when they presented specifically for sexual health advice, and were most likely to test for STIs in a symptomatic presentation. They were less comfortable discussing sexual health opportunistically; most did so only sporadically.

Generally, GPs had a reactive rather than proactive approach to sexual health promotion. In keeping with other research suggesting sexual health consultations may be difficult for both the GP and patient, ${ }^{17}$ GPs voiced the challenge of finding an appropriate segue into the subject of sexual health for a young man. In stark contrast to previous findings, ${ }^{5-7}$ some GPs believed that young men would find discussion of their sexual health to be confronting.

GPs identified several barriers to promoting sexual health, also previously reported in international studies. ${ }^{11,18,19}$ Many GPs mentioned lack of time and their heavy workload, which make it difficult to discuss anything preventive, let alone sexual health. Other barriers that GPs perceived would limit young men's receptiveness to sexual health discussions included embarrassment, gender and age discordance and a lack of sexual knowledge on the young man's part.

Young men having fewer interactions than young women in primary care limits the possibility of opportunistic screening for STIs and other preventive health measures. This infrequent interaction appeared to compound the uncertainty about whose responsibility it is to raise the issue of sexual health in an unrelated consultation. Many GPs saw it as their responsibility to ask about a young man's sexual health whenever possible, with most GPs believing sexual health to be a shared responsibility between young men and their GPs. Young men were seen as having the primary responsibility to seek advice if they noticed symptoms or changes or were concerned about an issue. This demonstrates a mismatch in expectations between some GPs and young men, as our previous research has shown that young men would like GPs to initiate a sexual health discussion independent of the type of presentation. ${ }^{5}$ Young men are generally poorly informed about sexual health, and while they may seek information from a variety of sources such as family, the internet, friends and pornography, they see most of these sources as unreliable. ${ }^{6}$ GPs are seen as a trusted source but are often not accessed because of embarrassment. ${ }^{6}$

GPs can help young men feel more comfortable by affirming privacy and acknowledging that while sexual health is a sensitive topic, it is a very important issue. It is likely that single promotional strategies will not be enough, and multiple methods of promotion are more likely to best reach and engage young men., ${ }^{77}$

GPs and other primary care clinicians are well placed to discuss sexual health with young men and thereby contribute to controlling the increasing rates of many STIs. Our results suggest that some GPs may benefit from additional support around initiating sexual health consultations. For example, the Red Book could be used to provide more detail about what is needed for sexual health promotion, ${ }^{13}$ and could also offer some detail on how GPs could initiate a sexual health discussion.

\section{Strengths and limitations}

The sample in this study was drawn from GPs who were willing to be interviewed; those not willing to participate in this study may have provided different answers. Snowball sampling was partially used for recruitment and may have caused some sampling bias, although the use of a sampling framework to ensure participants met a broad range of characteristics countered this. Interviews were undertaken with GPs in metropolitan, rural and regional Victoria. Male and female GPs with varied levels of interest in sexual health were included. The strength of this study was the use of a qualitative method to explore this sensitive issue and provide in-depth views, the frequency of which can be determined in a future quantitative work.

\section{Conclusions}

This study used qualitative research to explore the attitudes of GPs in relation to promoting sexual health to young men. Generally, GPs had favourable attitudes towards managing a young man's sexual health but did not always see it as their responsibility to initiate this discussion. This is at odds with young men's views, as shown in previous research. GP initiation of sexual health discussion is likely to highlight to young men the importance of maintaining sexual health, and many young men would welcome this.

Our findings suggest that sexual health promotion to young men in the general practice setting is best conducted within a preventive health framework. Interventions to improve GPs' confidence and sense of responsibility concerning managing a young man's sexual health could lead to increased safe sex messages to young men and therefore safer sex practices. Professional development in sexual health may also assist. Letting GPs 
Table 1: GP characteristics

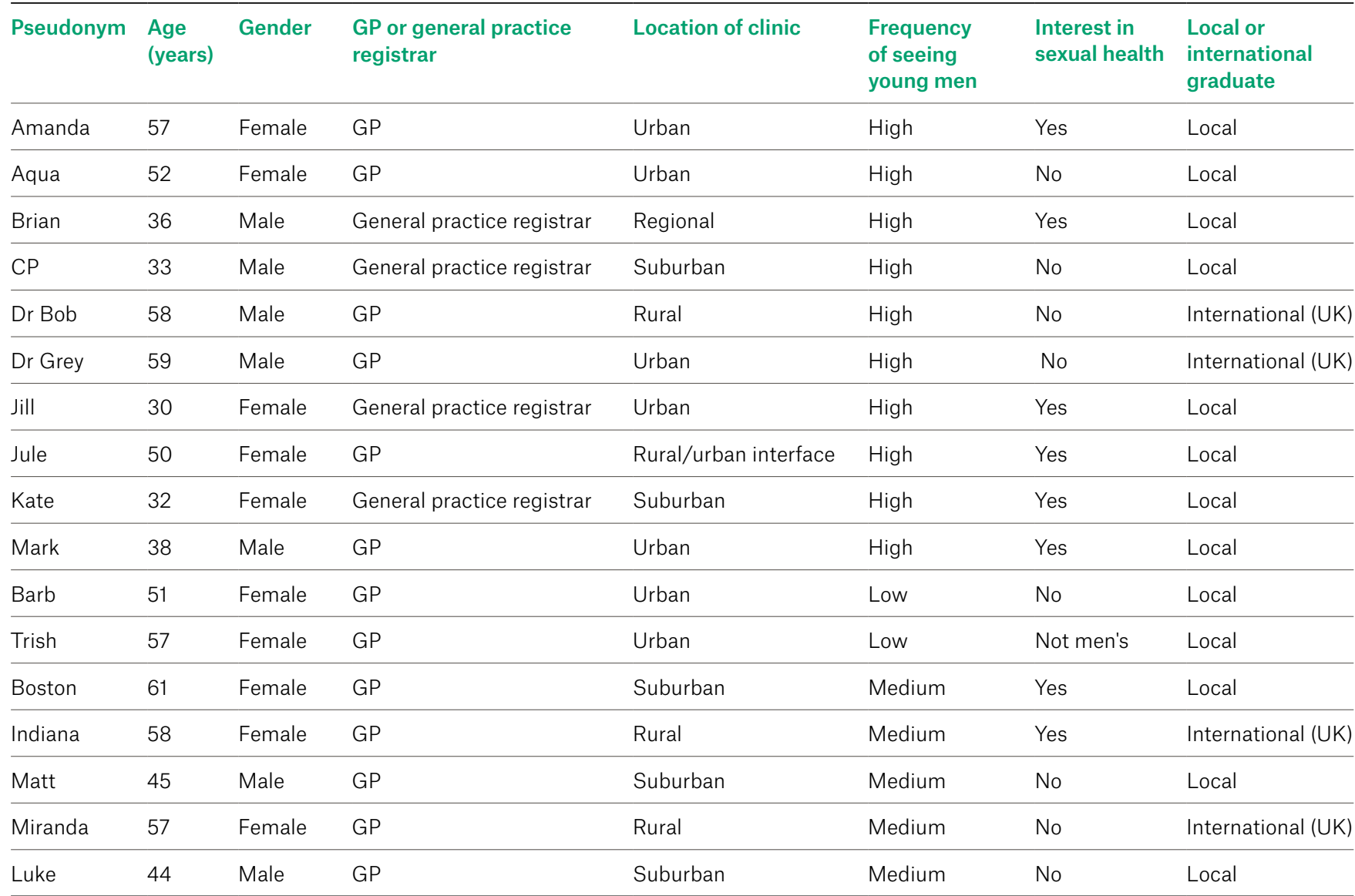

Demographics of GPs including their pseudonym, age, gender, whether they are a GP or GP registrar, the location of their clinic, frequency of seeing young men, the GPs' interest in sexual health and whether they were local or international medical graduates.

GP, general practitioner

High: daily, medium: weekly, low: less than once a week

know that young men are happy to receive sexual health promotion is likely to be an important facilitator.

\section{Implications for general practice}

- Some GPs see it as a young man's responsibility to initiate a sexual health discussion, but GPs should be aware that their young male patients may be waiting for the GP to raise this.

- Affirming privacy and reassuring young men of the legitimacy and importance of discussing sexual health is likely to be welcomed by young men.

\section{Authors}

Archibald Collyer MD, BSc (Hons), Resident, Northeast Health, Wangaratta, Vic; Department of General Practice, University of Melbourne, Vic. Archibald. collyer@gmail.com
Siobhan Bourke MBBS, FAChSHM, MPH, GradCert $\mathrm{PH}$ (Sexual Health), Senior Lecturer, Centre for Excellence in Rural Sexual Health, University of Melbourne, Vic.

Meredith Temple-Smith DHSc, MPH, BSc, Professor and Director, Research Training, General Practice, University of Melbourne, Vic.

Competing interests and funding: None.

Provenance and peer review: Not commissioned, externally peer reviewed.

\section{Acknowledgements}

The researchers would like to thank the GPs involved in the study and VicReN and CERSH for assisting in the recruitment of GPs.

\section{References}

1. de Visser RO, Badcock PB, Rissel C, et al. Safer sex and condom use: Findings from the Second Australian Study of Health and Relationships. Sex Health 2014;11(5):495-504.

2. Mitchell A, Patrick K, Heywood W, Blackman P, Pitts M. 5th National Survey of Australian Secondary Students and Sexual Health 2013. Melbourne: Australian Research Centre in Sex Health and Society, La Trobe University, 2014.
3. Kong FY, Guy RJ, Hocking JS, et al. Australian general practitioner chlamydia testing rates among young people. Med J Aust 2011;194(5):249-52.

4. Britt H, Miller GC, Henderson J, et al. General practice activity in Australia 2015-16. General Practice Series no. 40. Sydney: Sydney University Press, 2016.

5. Latreille S, Collyer A, Temple-Smith M. Finding a segue into sex: Young men's views on discussing sexual health with a GP. Aust Fam Physician 2014;43(4):217-21.

6. Litras A, Latreille S, Temple-Smith M. Dr Google, porn and friend-of-a-friend: Where are young men really getting their sexual health information? Sex Health 2015;12(6):488-94.

7. Ewert C, Collyer A, Temple-Smith M. 'Most young men think you have to be naked in front of the GP' A qualitative study of male university students views on barriers to sexual health. Sex Health 2016;13(2):124-30.

8. Chaudhary R, Heffernan CM, Illsley AL, et al. Opportunistic screening for chlamydia: A pilot study into male perspectives on provision of chlamydia screening in a UK university. J Public Health (Oxf) 2008;30(4):466-71. 
9. Lau A, Spark S, Tomnay J, et al. Sociodemographic and structural barriers to being tested for chlamydia in general practice. Med $\mathrm{J}$ Aust 2016;204(3):112.e1-5.

10. Poljski C, Tasker C, Andrews C, Wijesinha S, Piterman L, de Kretser D. GP attitudes to male reproductive and sexual health education and promotion. A qualitative study. Aust Fam Physician 2003;32(6):462-5.

11. Marcell AV, Ellen JM. Core sexual/reproductive health care to deliver to male adolescents: Perceptions of clinicians focused on male health. J Adolesc Health 2012;51(1):38-44.

12. Macdowall W, Parker $R$, Nanchahal $K$, et al. 'Talking of Sex': Developing and piloting a sexual health communication tool for use in primary care. Patient Educ Couns 2010;81(3):332-37.

13. The Royal Australian College of General Practitioners. Guidelines for preventative activities in general practice. 9th edn. Melbourne: RACGP, 2016.

14. Cummings $M$, Kang $M$. Youth health services: Improving access to primary care. Aust Fam Physician 2012;41(5):339-41.
15. Lewis-Beck M, Bryman A, Futing Liao T. The Sage encyclopedia of social science research methods. London: Sage, 2004.

16. Wainwright $M$, Russell A. Using NVivo audiocoding: Practical, sensorial and epistemological considerations. Social Research Update 2010(60):1.

17. Woodbridge MR, Dowell AC, Gray L. 'He said he had been out doing the traffic': General practitioner perceptions of sexually transmitted infection and HIV testing strategies for men. J Prim Health Care 2015;7(1):50-56.

18. Lorimer K, Martin S, McDaid LM. The views of general practitioners and practice nurses towards the barriers and facilitators of proactive, internetbased chlamydia screening for reaching young heterosexual men. BMC Fam Pract 2014;15:127.

19. Wimberly $Y H$, Hogben M, Moore-Ruffin J, Moore SE, Fry-Johnson Y. Sexual history-taking among primary care physicians. J Natl Med Assoc 2006;98(12):1924-29. 